\title{
Las partículas subjetivas del español centroamericano: indagaciones preliminares ${ }^{1}$
}

\section{(Subjective Particles in Central American Spanish: A Preliminary Inquiry)}

\author{
Viviana Núñez Alvarado² \\ Universidad Nacional, Costa Rica
}

\begin{abstract}
Resumen
Se plantea una etapa preliminar para elaborar un inventario de palabras con el perfil de «partícula». El estudio corresponde al interés en analizar una clase de palabras marginal no solo en cuanto a la tipología del español, sino también en investigaciones en lingüística hispánica. Se establece el marco referencial y conceptual para el estudio y se presenta el inventario inicial de recopilación y tabulación.
\end{abstract}

\begin{abstract}
This preliminary inquiry consists of an inventory of words with "particles," as defined here. The study corresponds to the interest of analyzing a marginal type of words according not only to Spanish typology, but also to Hispanic linguistic research. The referential and conceptual framework is provided for this study, as well as the inventory itself up to the latest collection and tabulation.
\end{abstract}

Palabras clave: tipología del español, español centroamericano, partículas subjetivas, español.

1 Recibido: 16 de abril de 2018; aceptado: 17 de setiembre de 2018.

2 Facultad de Filosofía y Letras, Universidad Nacional, Costa Rica. Correo electrónico: viviana. nunez.alvarado@una.cr

LETRAS 64 (2018), ISSN 1409-424X; EISSN 2215-4094

Doi: http://dx.doi.org/10.15359/rl.1-64.8

www.revistas.una.ac.cr/index.php/letras 
Keywords: Spanish typology, Central American Spanish, subjective particles

En esta indagación preliminar se plantea un marco referencial y conceptual referido a las partículas, desarrollado en Quesada ${ }^{3}$, con el que se aborda el estudio preliminar de un inventario representativo de palabras que se consideran partículas según ese marco referencial adoptado.

\section{Definición y delimitación}

El término partícula se ha utilizado sobre todo en lenguas en las que este tipo de palabras desempeña un papel marginal para subsumir palabras tan diversas como interjecciones, adverbios, o conjunciones. El criterio que subyace a ese proceder, según Quesada, es que todas esas palabras tienen en común la falta de flexión. El resultado es una clase heterogénea de palabras; y lleva a largas listas de «partículas» en muchos idiomas. Afirma que «desde la perspectiva de una teoría general de partículas tal procedimiento tiene serias debilidades; baste con mencionar que el criterio de flexión no es confiable pues no es aplicable a todas las lenguas» (149); en lenguas aislantes, por ejemplo. Por ello, Quesada adopta la definición de Wedyt y Hentschel (1989), quienes dividen los significados de las formas lingüísticas en léxicos (o categoremáticos), deícticos, partes de la oración y sincategoremáticos, los cuales generan tres grupos de palabras:

a. principales (sustantivos, adjetivos, verbos), con significado léxico y con referentes como entidades, eventos y características; b. palabras que no refrieren a la realidad externa como los deícticos, pronombres

3 Juan Diego Quesada, «De ahi > diay: A Particle Is Born. Discourse-Triggered Grammaticalization in Spanish», Zeitschrift für Sprachwissenschaft 15, 2 (1996): 147-177. DOI: https://doi. org/10.1515/zfsw.1996.15.2.147. [El marco referencial se ha fundamentado principalmente del estudio de Quesada; la versión en español de las citas y de los términos particulares es responsabilidad de la autora del presente artículo. En adelante los números de página se indican entre paréntesis en el texto (N. de la E.)]. 
y las pro-formas en general, y c. palabras sin significado léxico o deíctico y cuya semántica se obtiene solo en relación con otras partes de la oración (149).

Las partículas son ejemplo de este grupo; por ello se definen como palabras sin significado léxico o de parte de la oración. Esta división de Wedyt y Henstschel se representa gráficamente en el esquema 1.

Esquema 1.

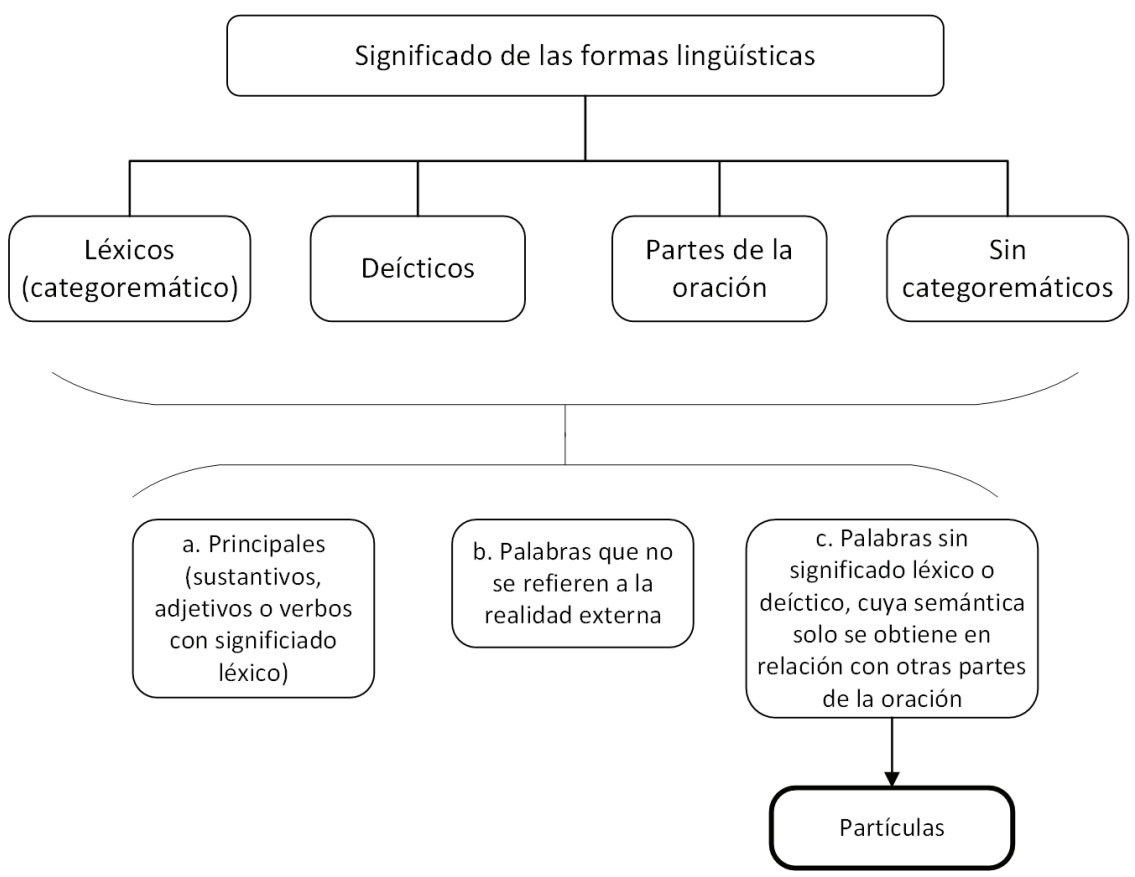

Wedyt y Hentschel, según lo planteado por Quesada:

delimitan las partículas en contraposición a palabras similares como los adverbios y las interjecciones. Según los autores, los adjetivos predicativos no califican como partículas en virtud de su capacidad para referenciar características que existen en la realidad externa, así 
como por sus rasgos distribucionales especiales. Lo mismo vale para adverbios de lugar (aquí, allá) y de modo. El comportamiento de los adverbios refleja la naturaleza variable de esta clase de palabras, lo cual en última instancia dificulta categorizarlos como sincategoremáticos. Por ello, señalan que casos intermedios en los que el asignar una palabra a una u otra clase se hace difícil (149).

\section{Agrega Quesada:}

Las otras palabras contra las cuales se debe delimitar el término partícula son las interjecciones, las cuales Schachter (1985: 58) define como «palabras, por le general de carácter exclamativo, que pueden constituir expresiones en sí mismas y que a menudo no tienen conexión sintáctica con otras palabras con las que co-aparecen» ${ }^{4}$. Weydt y Hentschel (1989) las excluyen de la categoría de partículas «porque con plena seguridad no pertenecen a la clase de palabras sincategoremáticas». Los tres tipos de interjecciones identificados por Weydt y Hentschel (1989) — las de tiras cómicas, onomatopéyicas y emocionales - tienen una referencia directa con la realidad externa, según esos autores. (149)

\section{Clasificación}

Partiendo de esa definición de partícula, Weydt y Hentschel (1989), según lo desarrollado por Quesada (150), proponen la siguiente clasificación:

1. Conjunciones: coordinantes y subordinantes; su función es la de unir oraciones.

2. Preposiciones: establecen relaciones y dividen la oración en partes; unen elementos nominales.

4 Paul Schachter, «Parts of Speech Systems», Timothy Schopen, ed., Language Typology and Syntactic Description, vol. 1 (Cambridge: Cambridge University Press, 1985) 3-61. 
3. «Partículas gradativas»: los autores usan el término para referirse a dos tipos de partículas (en realidad reconocen que el término es inadecuado)

3.1 Intensivas: modifican una característica expresada por otra palabra (por lo general un adjetivo) ya reforzándolo, ya debilitándolo, como ejemplos dan very del inglés y ziemlich del alemán.

3.2 De foco: forman un constituyente con el elemento que modifican, asignándole el estatus de foco; ejemplos de ello son ausgerechnet del alemán o solo del español.

4. Modales; expresan el grado de probabilidad de una expresión (por ejemplo, maybe del inglés ovielleicht del alemán. Dada su función de expresar probabilidad, estas partículas básicamente aparecen en oraciones enunciativas (es imposible que aparezcan en construcciones imperativas o que expresen deseo).

5. De matiz: el alcance de estas es extra-oracional; funcionan al nivel discursivo. De ellas los autores dicen que «comentan la oración como una expresión total desde un meta-nivel y la anclan de esa forma en el contexto del habla» (Weydt y Hentschel).

6. Pregunta y respuesta: niegan o afirman un estado de cosas o partes del mismo. (150)

«Esta clasificación muestra dos tipos de partículas dependiendo de su significado y función» (150). Quesada denomina «a las de 1., 2., algunas de 3. y a las de 6 como "fácticas" porque cumplen funciones sintácticas en esencia. Las conjunciones, las preposiciones, algunas partículas intensivas y algunas de foco simplemente llenan un espacio sintáctico en una secuencia; lo que tienen en común dice el autor es que su alcance es "local"» (150); es decir, "no va más allá de su entorno inmediato» (150). «Por el contrario, las de 4., 5. y 
algunas de 3. muestran un alcance extra-oracional y un uso subjetivo» (150); Quesada las denomina «subjetivas»; de ese modo, el uso de las partículas subjetivas depende del contexto y de la valoración del hablante sobre la situación ${ }^{5}$. El autor propone analizarlas como la gramaticalización ${ }^{6}$ de la categoría discursiva de VALORACIÓN; señala que en la literatura especializada se ha puesto especial atención en las partículas subjetivas por cuanto son esas las que generan mayor dificultad a los investigadores a la hora de dar cuenta de su significado y función de manera uniforme (150-151). Quesada cita el caso de diay como ejemplo de partícula que exhibe una serie de significados que a primera vista lucen diversos (151).

\section{Función de las partículas subjetivas}

«Las partículas subjetivas tienen una función eminentemente pragmática lo cual es tanto causa como consecuencia de su alcance amplio y oracional» (151). Otros, según Quesada, han considerado que «la función de las partículas subjetivas consiste en expresar tanto conocimiento como actitudes del hablante sobre una proposición» (151); Weydt (1989) las denomina «partículas de tonalidad» (151) porque matizan las expresiones; pueden expresar modo o estado de ánimo de la conversación cuando refieren a conocimiento compartido por los interlocutores. Quesada continúa:

5 Quesada (151, nota 3) aclara que desde un punto de vista general, el lenguaje es subjetivo; y que expresar oraciones es en sí mismo un asunto subjetivo. «No obstante, desde el punto de vista de la gramaticalización de actitudes, hay formas como los modales o marcadores de modo, cuya función principal es precisamente expresar ese aspecto, en oposición a otras formas del modo (realis), cuya función es básicamente proposicional».

6 Sobre gramaticalización puede consultarse: Bernd Heine, Grammaticalization: A Conceptual Framework (Chicago: Chicago University Press, 1991); Christian Lehman, Grammaticalization: Synchronic Variation and Diachronic Change. Lingua e Stile/a, 20, 3 (1985): 303-318; Christian Lehmann, Thoughts on Grammaticalization. A Pragmatic Sketch (Köln: Institut für Sprachwissenschaft der Universität, 1982); Elizabeth Traugott y Bernd Heine, eds., Approaches to Grammaticalization, 2 vols. (Amsterdam/Philadelphia: John Benjamins, 1991); Frantisek Lichtenbcrk, «Semantic Change and Heterosemy in Grammaticalization», Language 67 (1991): 475-509. DOI: https://doi.org/10.1353/lan.1991.0009; Paul). 
Según Abraham (1991: 333) ${ }^{7}$, las partículas subjetivas «hacen visible, mediante su fuerza ilocucionaria, lo que el hablante [...] considera adecuado para desambiguar totalmente cierto texto o porción discursiva precedente o subsecuente»; ello implica que las partículas subjetivas «detectan vacíos en la configuración argumentativa de un texto e indican parcialmente cómo el oyente o el lector deben reconstruir el argumento faltante». Corolario de esto es que la presencia de tales formas, siendo categoremáticas pero a la vez marcadores pragmáticos, depende de los tipos de oraciones y de la fuerza ilocucionaria, especialmente en situaciones informales. (151)

Quesada señala que, según Kirstein ${ }^{8}$, mediantelas partículas subjetivas «tanto hablante como oyente se indican mutuamente la "comprensión de sus roles" [comunicativos], sin necesidad de retomar el contenido lingüístico explícito (Kirstein 1983: 217)» (251). Quesada añade:

Partiendo de que el hablar crea situaciones, mientras que el hablar con partículas crea especialmente situaciones, Kirstein (1983: 217) señala que «las expresiones que contienen partículas, las cuales mediante una referencia explícita a ciertos factores situacionales como la actitud del hablante y comprensión del rol comunicativo, las que definen la situación para el oyente». Kirstein afirma que lo que define [el significado] de una partícula es el acto del habla; pues para él no tiene sentido hablar del significado primario de las partículas, o de si una partícula tiene un significado general que determine todas sus instancias. Si bien es cierto que la dependencia contextual es una característica de las partículas, ello no necesariamente implica que no se les pueda asignar un significado básico. (151-152)

\section{Quesada también se refiere a Foolen:}

Foolen (1989) distingue tres niveles de significado de las partículas: a. nivel más abstracto con el significado general del ítem léxico (...);

7 Werner Abraham, Discourse Particles (Amsterdam: Benjamins, 1991) 203-252.

8 Boni Kirstein, «Partikeln und Sprechsituation», Harald Weydt, ed., Partikeln und Interaktion (Berlín: De Gruyter, 1982) 213-225. DOI: https://doi.org/10.1515/9783111661643.213. 
b. nivel intermedio en el que el significado general también está presente pero solo en el uso de la partícula; c. nivel semántico concreto, en el que se especifica la semántica de los usos que se dan bajo el significado general; este último es en la práctica un nivel pragmático. Esto constituye un procedimiento metodológico apropiado tanto para la descripción sincrónica como la diacrónica, pues los tres niveles en realidad corresponden a etapas de gramaticalización. (152)

\section{Al respecto, Quesada también se refiere a Fábricz (1989) quien}

subraya la relación entre partículas y sus fuentes (por lo general conjunciones, adverbios o elementos deícticos) y llama la atención sobre el hecho de que «el significado de una partícula modal se origina en el significado de su étimo filtrado por la predicación», caracterización que resume las características de las partículas subjetivas; éstas son formas que resultan de la gramaticalización de diferentes tipos de palabras, cuyos significados contienen el material semántico necesario para generalizarse al nivel textual; ese material semántico incluye conceptos generales, tales como adición, oposición, causa, resultado, etc. (152)

\section{Partículas subjetivas en español}

\section{Quesada afirma:}

En la tradición filológica hispánica, el término partícula también se ha usado como paraguas para incluir palabras como interjecciones, adverbios, conjunciones, etc. (...) Las principales características que se les asignan a las partículas son su alcance extra-oracional y su valor modal o subjetivo. Es una equiparación implícita de partícula con partícula subjetiva; de hecho, las partículas fácticas pocas veces despiertan el interés de los gramáticos. (...) Salvá y Bello se referían a la gradación subjetiva de algunas partículas, con lo que implicaban la coexistencia de dos significados y funciones, uno «literal» y otro «subjetivo», con etapas intermedias entre ellos. Esta percepción corresponde a lo que la teoría de gramaticalización ahora 
denomina especialización y subjetivización de una forma gramaticalizada; la primera es la condensación de los matices semánticos de la forma respectiva en un dominio funcional; la escogencia cada vez más restringida de posibilidades adquiere significados gramaticales más generales (Hopper 1991); la segunda consiste en el cambio de significado de la forma que se gramaticaliza, la cual «se torna más centrada en la actitud del hablante sobre la situación» (Hopper y Traugott 1993) $)^{9}$ (153)

«Dado que la subjetivización no es un rasgo exclusivo de las partículas, sino que constituye un importante ingrediente de la gramaticalización» (153), Quesada no ve apropiado «subsumir otros tipos de palabras como adverbios o conjunciones bajo la clase de partículas porque la subjetivización por sí sola no es suficiente para analizar una forma como partícula» (153); pues es, siguiendo a Wedyt y Hentschel (1989), afirma Quesada:

su estatus sincategoremático lo que las define como tales. Es decir, es la mezcla de significado sincategoremático y significado subjetivo lo que hacen de una forma lingüística una partícula subjetiva. Esta delimitación es crucial para caracterizar una lengua (en este caso el español) que utiliza este tipo de palabras y de las categorías gramaticales que codifican. (153)

En este sentido, Quesada afirma que no se puede caracterizar al español como una lengua de partículas, como es el caso del alemán, el holandés el latín o el tzotzil (153). Ello no impide, al mismo tiempo, que se haga un inventario de las pocas partículas y se analicen; eso sí, teniendo presente que se trata de una clase marginal en la gramática española.

Quesada sigue:

Mientras que Bello le puso más atención al carácter subjetivo de las partículas (independientemente de cómo las definiera), Gili Gaya

9 Paul Hopper y Elizabeth Traugott, Grammaticalization (Cambridge: Cambridge University Press, 1993. 
(según Martín Zorraquino 1992) recalca su alcance extra-oracional como manifestación de la función textual de mantención de la coherencia, las cual se logra mediante la coordinación, la negación, la inferencia y la modalidad. Esa categoría está definida de manera tan amplia que hasta adverbios, frases adverbiales, fraseologismos e interjecciones son analizadas como partículas. (153)

Se puso de moda durante algún tiempo entre filólogos la noción de conectores pragmáticos, «cuyo común denominador», afirma Quesada, «es el alcance extra-oracional de las formas. Así pues, Solano (1990:7) justifica subsumir todo tipo de palabras bajo esa etiqueta por el hecho de que establecen lazos entre oraciones» (153). Al respecto, Quesada cita a Solano:

Desde luego, un texto o discurso no es una secuencia casual o desordenada de oraciones relacionadas por el contenido. Hay dispositivos formales que señalan la naturaleza de esas relaciones. Esos enlaces o recursos de conexión pueden ser gramaticales (nexos), léxicos, o entonacionales en el caso del habla y tienen como función fundamental "conectar" oraciones y secuencias de oraciones ${ }^{10}(153)$.

Quesada critica este procedimiento onomasiológico pues tiene la «desventaja de desconocer el estatus formal de los ítems lingüísticos y su papel en el sistema general de la lengua» (154). Además, argumenta que: «el término "conexión" es tan etiqueta de funciones tanto como partícula lo es de formas, sin mencionar el hecho de que algunas partículas subjetivas en algunas funciones no conectan nada, sino que expresan la valoración subjetiva de una proposición» (154); y de esa manera se eclipsa la función exacta de las nociones o conceptos que se gramaticalizan.

10 Yamileth Solano, Los nexos en el habla culta costarricense (Mexico: UNAM, 1990) 7. 


\section{Partículas subjetivas en Centroamérica ${ }^{11}$}

Con base en lo anterior, se ha realizado un estudio preliminar a partir de un corpus específico. Los resultados arrojan seis partículas subjetivas principales: verdad, pues, bueno, claro, vaya y diay. Dos de ellas, diay y verdad, y en menor grado pues, presentan variantes caracterizadas por erosión fonológica, cuyas implicaciones están pendientes para el trabajo posterior. Estas partículas no tienen distribución homogénea en el istmo centroamericano (ver anexo); algunas de ellas son más frecuentes en algunas zonas que en otras, mientras que otras ni siquiera se utilizan en algunos lugares. Ejemplos de ello son verdad y sus variantes, las cuales se distribuyen por todo el norte (Guatemala, El Salvador, y Honduras), mientras que diay es casi exclusiva de Costa Rica e inexistente en el norte.

En cuanto al género, el corpus utilizado incluyó tres hombres y tres mujeres por país, por lo que en los gráficos (ver anexo) se indica cuántas veces apareció la partícula en cada caso y el porcentaje correspondiente. El orden de presentación está determinado cuantitativamente; es decir, según la cantidad de tokens que hay en el corpus.

Los gráficos (ver anexo) muestran la frecuencia con que aparece cada partícula en el corpus analizado. El corpus corresponde al texto El español de Centroamérica: materiales para su estudio, de Herrera, Nuñez y Quesada (desarrollado en 2012) ${ }^{12}$, que transcribe grabaciones hechas a seis personas por país centroamericano, de diferente nivel educativo y tomando en cuenta ambos géneros, es decir, en un

11 Para más información sobre el español de Costa Rica en particular pueden consultarse también los siguientes textos: Carlos Gagini, Diccionario de costarriqueñismos (San José: Editorial Costa Rica, 1892/1975); Miguel Ángel Quesada, Fuentes documentales para el estudio del español de Costa Rica (San José: Alma Mater, 1987); Miguel Ángel Quesada, El español colonial de Costa Rica (San José: Editorial de la Universidad de Costa Rica, 1990); Miguel Ángel Quesada, Nuevo diccionario de costarriqueñismos (Cartago: Editorial Tecnológica, 1991); Miguel Ángel Quesada, Diccionario histórico del español de Costa Rica (San José: EUNED, 1995); Víctor Manuel Arroyo, El habla popular en la literatura costarricense (San José: Universidad de Costa Rica, 1971).

12 Al respecto, ver Gisselle Herrera Morera, Viviana Núñez A. y J. Diego Quesada, El español de Centroamérica. Visión global y materiales para su estudio, 2 tomos (Heredia: Editorial Universidad Nacional, 2016). 
total se entrevistó a 36 personas. El «total» indicado en cada gráfico corresponde a la cantidad de veces que aparece utilizada la partícula por los informantes; cada país se representa con un color diferente y se indica entre paréntesis las veces que esa partícula se utilizó en ese país. También se indica el porcentaje correspondiente.

En relación con la variable «edad», en el gráfico correspondiente al grupo etario (ver anexo), el número 1 corresponde a informantes cuya edad oscila alrededor de los veinte años; el número 2, a informantes de unos treinta y cinco años; y el número 3 , a adultos de sesenta años aproximadamente.

Este trabajo es meramente de inventario; no se realiza ningún análisis de significados y funciones, el cual queda pendiente.

Verdad: Esta partícula es utilizada mayoritariamente en el triángulo norte de Centroamérica (Guatemala, El Salvador y Honduras); constituye el $85 \%$ de la muestra. La partícula es utilizada principalmente por hombres y dentro de esta variable su distribución tiende a ser equitativa entre los tres grupos etarios. En términos generales, su utiliza con una función de reafirmación ${ }^{13}$ (ver anexo: gráfico 1).

Pues: Partícula utilizada mayoritariamente por hombres jóvenes, es frecuente en Guatemala y en Nicaragua. No se le puede adscribir una única función, pues parece tener diversos grados de gramaticalización, los cuales redundan en una variedad de funciones que deben ser analizadas en detalle (ver anexo: gráfico 2).

Bueno: Es utilizada principalmente en Honduras y en El Salvador, y después por Costa Rica y Guatemala. Principalmente se registra entre por jóvenes. No se registra mayor diferencia en el género. Tiende a usarse para diversas funciones comunicativas tales como ilativa y afirmativa (ver anexo: gráfico 3).

13 Dado que el objetivo de este trabajo es hacer un inventario de las partículas de la muestra, solamente se atribuyen funciones a estas formas de manera muy superficial. Un análisis pormenorizado de las funciones queda para una investigación posterior. 
Claro: Utilizada mayoritariamente en Costa Rica, puede definirse junto con diay como partículas costarricenses por excelencia. Principalmente la utilizan personas del segundo grupo etario; la variable género no presenta grandes diferencias. Se utiliza principalmente para expresar acuerdo con el punto de vista del oyente (ver anexo: gráfico 4).

Vaya: Se utiliza sobre todo en El Salvador; es más frecuente entre mujeres del segundo grupo etario. Pareciera utilizarse principalmente para expresar acuerdo (ver anexo: gráfico 5).

Diay: Es una partícula utilizada mayoritariamente en Costa Rica. En la muestra la utilizan sobre todo por hombres del primer grupo etario (ver anexo: gráfico 6). Esta partícula ya ha sido analizada por Quesada (1996, 156-173).

\section{Conclusión}

Este inventario de partículas subjetivas del español en Centroamérica arroja datos valiosos, independientes de un análisis de significados y funciones, como son la distribución geográfica, por género y por edad. Martín Zorraquino ${ }^{14}$ advertía que las partículas en español son indicadoras de rasgos diatópicos, diastráticos y diafásicos. El caso de diay es un claro ejemplo de ello; es, como lo indica Quesada, la partícula de Costa Rica por excelencia (este estudio revela que un estatus similar lo tiene claro), al igual que vaya lo es de El Salvador y verdad del triángulo norte de Centroamérica. Si se toma en cuenta que es entre hombres jóvenes en que predomina, se puede concluir que es una forma saludable y con una función sociolingüística como factor de identidad. Lo mismo se puede decir sobre las demás partículas, cuyo análisis detallado queda pendiente para futuras investigaciones.

14 María Antonia Martín Zorraquino, «Spanisch: Panikel forschung/Partículas y modalidad», Lexikon der Romanistischen Linguistik VI, 1 (1992): 110-124 (112). 


\section{Anexo}
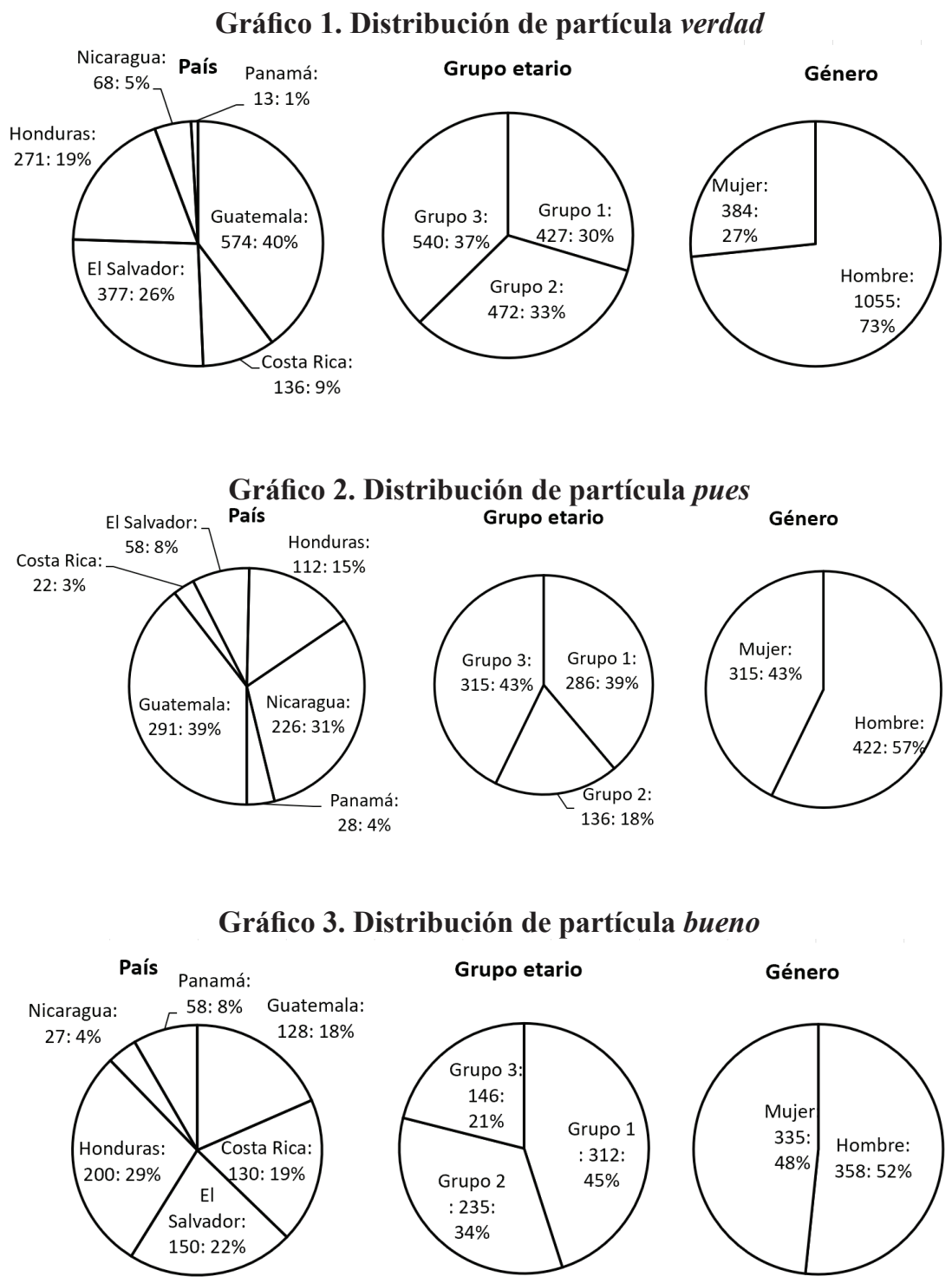

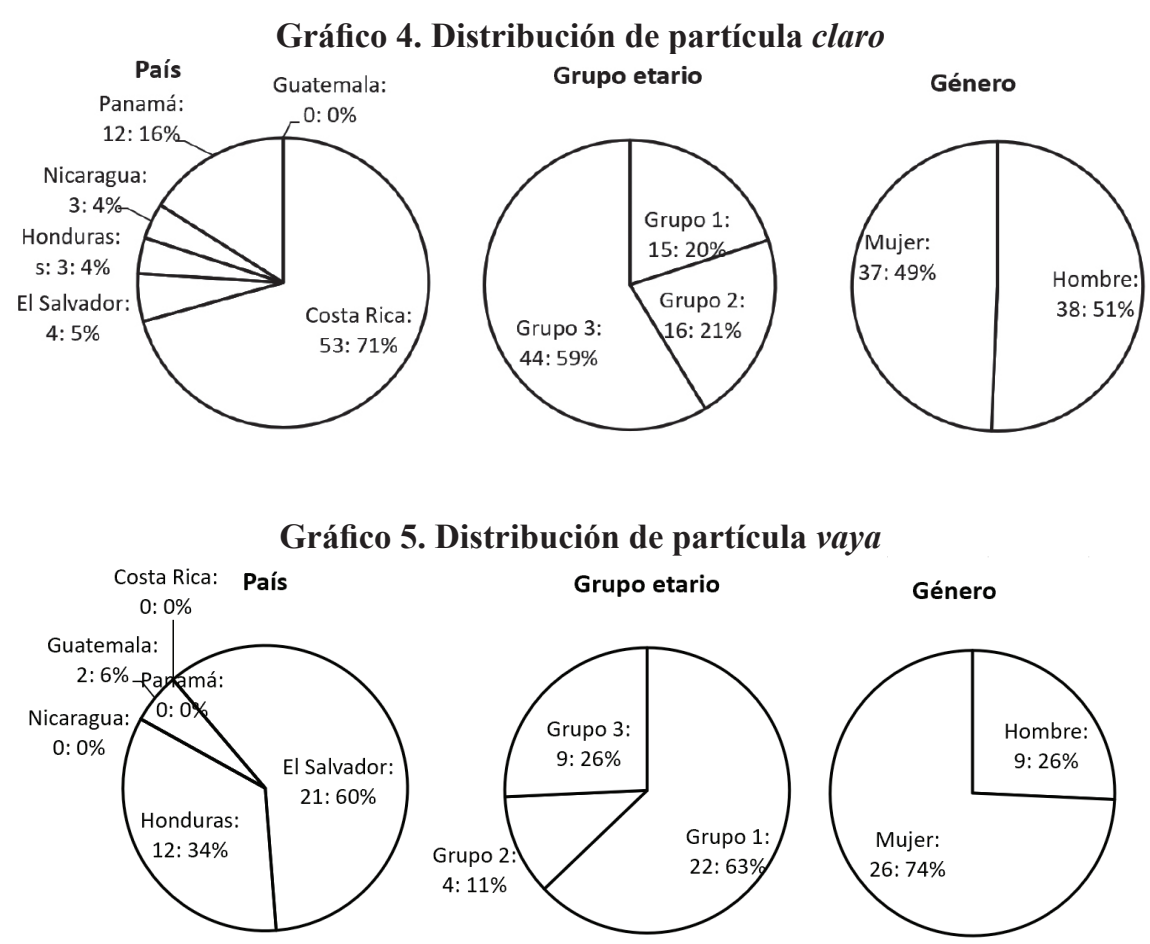

Gráfico 6. Distribución de partícula diay

País

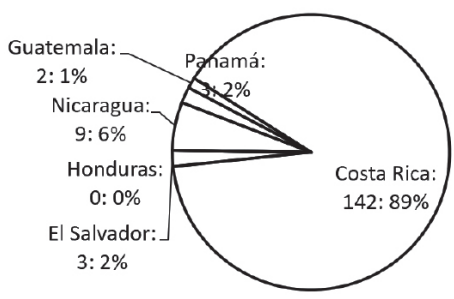

Grupo etario

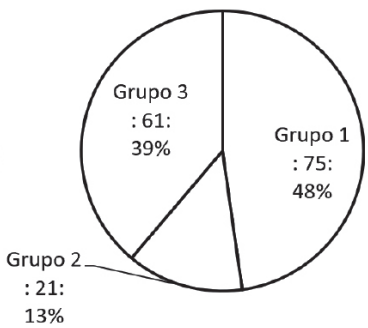

Género

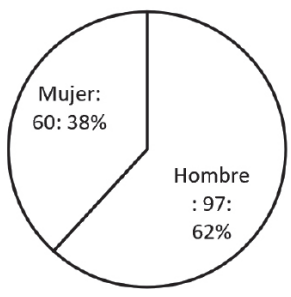


